\title{
HIGHER OBSTRUCTIONS TO SECTIONING A SPECIAL TYPE OF FIBRE BUNDLES
}

\author{
BY \\ WU-CHUNG HSIANG(1)
}

Introduction. The purpose of this paper is to investigate the problem of higher obstructions to sectioning a certain type of fibre bundles. Let $F$ be the fibre of a given bundle $\xi$, and let $n$ be the least dimension such that $\pi_{n}(F)$ is not zero. Then, the primary obstruction cocycles to sectioning $\xi$ form a unique class $O_{1}(\xi) \in H^{n+1}\left(X ; \pi_{n}(F)\right)$ where $X$ is the base space of $\xi$ [18]. If $m$ is the next dimension such that $\pi_{m}(F)$ is not zero and $O_{1}(\xi)=0$, then the secondary obstruction cocycle generally do not form a single class but a set of classes in $H^{m+1}\left(X ; \pi_{m}(F)\right)$. When $m \leqq 2 n$, there are general formulas to relate these secondary obstruction classes $[6 ; 9]$. It is relatively difficult to describe the relationship of these secondary obstruction classes when $m>2 n$. In this paper, we shall study the higher obstructions to sectioning a special type of fibre bundles such that $\pi_{2}(F)$ is the first nontrivial homotopy group of the fibre and the dimension of the next homotopy group of $F$ is much higher than 4 .

Let $F$ be a homogeneous space of a compact connected semisimple Lie group $G$. Suppose that the first nontrivial homotopy group of $F$ is $\pi_{2}(F)$ which is free. Let $\xi$ be a bundle over $X$ with fibre $F$ and group $G$ and whose primary obstruction vanishes. We will construct a principal torus bundle $F_{1} \rightarrow F$, a central extension $G_{1}$ of $G$ by the torus, and a set of weakly associated bundles $\{\zeta\}$ over $X$ with fibre $F_{1}$ and group $G_{1}$. Under the projection $p: \xi \rightarrow \zeta$, secondary and tertiary obstructions for $\xi$ corresponding to primary and secondary obstructions for $\zeta$ 's (cf. Theorem (4.3)). We shall call this method enlarging the fibre and the group. Thus, an enlargement may be used to reduce the order of an obstruction.

By this method, we rederive Kundert's formula for the secondary obstructions when the fibre is a complex projective space $C P^{n-1}$ and $P U(n)$ is the structural group $\left({ }^{2}\right)$. We will also derive new formulas for the third obstructions when $F=C P^{n-1}$ and for the secondary obstructions $\bmod 2$ to finding an orientable 2plane sub-bundle of an orientable vector space bundle (cf. Theorems (6.1) and (7.1)).

The author extends his cordial thanks to Professor N. E. Steenrod for his constant encouragement, suggestions and criticisms. He equally thanks Professors J. C. Moore, J. W. Milnor and Dr. P. F. Baum for many helpful discussions.

Received by the editors January 8, 1963.

(1) This paper was taken from a part of the author's thesis submitted to Princeton University in September, 1962.

(2) Actually, Hirzebruch [5] implicitly used this method to rederive Kundert's formula. 
I. Preliminary definitions and notations. In this paper we assume all the space under consideration are in the category $z$ of arcwise connected countable $\mathrm{CW}$ complexes with definite base points.

Let $F$ be a space, and let $G$ be a topological group acting on $F$. We shall call $(F, G)$ a fibre pair. For any space $X$ in $\jmath, \mathbf{B}(X ; F, G)$ denotes the set of equivalence classes of fibre bundles over $X$ with fibre $F$ and group $G$ [17]. If $f: X \rightarrow Y$ is a map of spaces, then it induces a map $f^{*}: \mathbf{B}(Y ; F, G) \rightarrow \mathbf{B}(X ; F, G)$ which sends $\xi \in \mathbf{B}(Y ; F, G)$ to $f^{*} \xi$. The transformation $T$ defined by $T(X)=\mathbf{B}(X ; F, G)$, $T(f)=f^{*}$ is a contravariant functor from $z$ into the category $\mathfrak{w}$ of sets. The image of $z$ under $T$ is a subcategory of $\mathfrak{w}$. We will call this subcategory $\mathbf{B}$, the category of fibre bundles (with fibre $F$ and group $G$ ).

For convenience, we list the following notations.

Greek letters, e.g., $\xi, \eta, \zeta, \cdots$ will denote fibre bundles.

$v(F, G)$ denotes the universal bundle with fibre $F$ and group $G$. We abbreviate it as $v$ if no confusion arises.

We use standard notations for the classical groups: $\mathrm{U}=$ unitary, $\mathrm{SO}=$ special orthogonal, $\mathrm{PU}=$ projective unitary $=\mathrm{U} /$ centre and $\mathrm{PSO}=\mathrm{SO} /$ centre. In every case a dimensional index is understood, $\mathrm{U}=\mathrm{U}(n)$, and is omitted when no confusion arises. The group $\mathrm{SO}(2)$ will be also denoted by $\mathrm{C}$ (for circle).

We use the standard notation $K(\pi, n)$ for an Eilenberg-Maclane space( $\left.{ }^{3}\right)$.

If $f: G_{1} \rightarrow G_{2}$ is a group homomorphism, then it induces a unique mapping up to homotopy of the classifying spaces $B\left(G_{1}\right) \rightarrow B\left(G_{2}\right)$. This map will be denoted by $f(B)$.

II. Triad of groups. In this section, we shall study a special problem on groups. Since it is only applied to special cases, we will state our definition and theorems in a minimal generality. All groups appearing in this section are compact connected Lie groups.

2.1. Definition. A triad of groups $\left(G_{1} ; T, H\right)$ consists of a group $G_{1}$, a central subgroup $T$ (hence a torus), and a subgroup $H$ such that $T \cap H=1$.

With any triad of groups $\left(G_{1} ; T, H\right)$ there is associated the following diagram which will be called the canonical diagram of the triad:

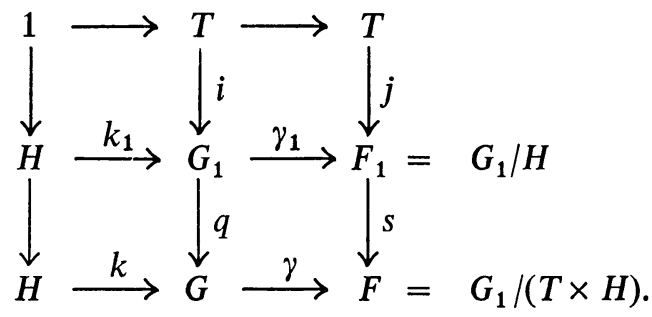

(3) $K(\pi, n)$ is an abelian group under Milnor's geometric realization [13] provided that $\pi$ is countable. 
Each row and column of the canonical diagram of a triad is a principal fibre bundle. Any group pair $(G, H)$ such that $H$ is a subgroup of $G$ is a triad by considering $T=1$.

2.2. Definition. We say that two triads $\left(G_{1} ; T, H\right)$ and $\left(G_{1}^{\prime} ; T^{\prime}, H^{\prime}\right)$ are equivalent if there is an isomorphism $f: G_{1} \rightarrow G_{1}^{\prime}$ such that $f(T)=T^{\prime}, f(T \times H)=T^{\prime} \times H^{\prime}$.

Note that we do not require $f(H)=H^{\prime}$ in 2.2. But $f(T)=T^{\prime}$ and $f(T \times H)=T^{\prime} \times H^{\prime}$ imply $f(H) \cong H^{\prime}$.

Given a triad of groups $\left(G_{1} ; T, H\right)$, there are two fibre pairs $\left(F_{1}=G_{1} / H, G_{1}\right)$ and $\left(F=G_{1} /(T \times H), G=G_{1} / T\right)$ associated with it. For convenience, we adopt the following definition.

2.3. Definition. Given a triad $\left(G_{1} ; T, H\right),\left(F_{1}=G_{1} / H, G_{1}\right)$ and $\left(F=G_{1} /(T \times H)\right.$, $\left.G=G_{1} / T\right)$ will be called the larger pair and the smaller pair of $\left(G_{1} ; T, H\right)$, respectively.

The typical example of a triad is $(\mathrm{U}(n) ; \mathrm{C}, \mathrm{U}(n-1))$ where $\mathrm{C}$ is the centre of $\mathrm{U}(n)$ which is isomorphic to the circle group. The canonical diagram of $(\mathrm{U}(n) ; \mathrm{C}, \mathrm{U}(n-1))$ is

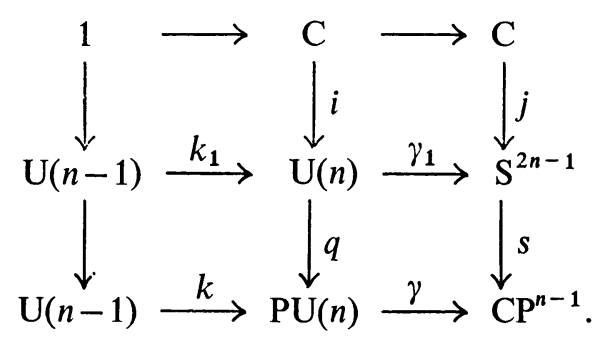

The larger pair of $(\mathrm{U}(n) ; \mathrm{C}, \mathrm{U}(n))$ is $\left(\mathrm{S}^{2 n-1}, \mathrm{U}(n)\right)$ and the smaller pair of $(\mathrm{U}(n) ; \mathrm{C}, \mathrm{U}(n))$ is $\left(\mathrm{CP}^{n-1}, \mathrm{PU}(n)\right)$.

Now suppcse given a fibre pair $(F, G)$ such that $F$ is a coset of $G$ relative to a subgroup $H$. Let $T$ be a torus. There always exists a triad $\left(G_{1} ; T_{1}, H_{1}\right)$ such that $T_{1} \cong T, H_{1} \cong H$ and $\left(G_{1} ; T_{1}, H_{1}\right)$ has $(F, G)$ as its smaller pair. In fact, we may simply let $G_{1}=T \times H, T_{1}=T \times e$ and $H_{1}=e^{\prime} \times H$. Obviously, there are many triads verifying these conditions. So, we need some invariants to characterize a triad.

Consider the last column of the canonical diagram (1) of a given $\operatorname{triad}\left(G_{1} ; T, H\right)$. It is a principal fibration

$$
T \rightarrow F_{1}=G_{1} / H \rightarrow F=G_{1} /(T \times H)
$$

with fibre torus $T$. Hence, the $k$-invariant of the fibration (3) is an element in $H^{2}\left(F ;(Z)^{m}\right)$ where $m$ denotes the rank of $T$.

2.4. Definition. The $k$-invariant of the fibration (3) which is the last column of the canonical diagram of the triad $\left(G_{1} ; T, H\right)$ is called the characteristic class of the triad and denoted by $k\left(G_{1} ; T, H\right)$. 
2.5. THEOREM. Suppose that $(F, G)$ is a fibre pair such that $G$ is a semisimple Lie group and $F$ is the coset space of $G$ relative to the subgroup $H$. Let $T^{m}$ be a torus of rank $m$ and $k$ be an element in $H^{2}\left(F ;(Z)^{m}\right)$. Then, there exists a unique triad $\left(G_{1} ; T_{1}, H_{1}\right)$ up to equivalence such that $T_{1} \cong T^{m},\left(G_{1} ; T_{1}, H_{1}\right)$ has $(F, G)$ as its smaller pair and $k\left(G_{1} ; T_{1}, H_{1}\right)=k$.

Proof. We first prove the existence part. Consider the following principal fibration:

$$
T^{m} \rightarrow F_{1} \rightarrow F
$$

with fibre $T^{m}$ and $k$-invariant $k \in H^{2}\left(F ;(Z)^{m}\right)$. Let $\gamma: G \rightarrow F$ be the projection. We have the following commutative diagram:

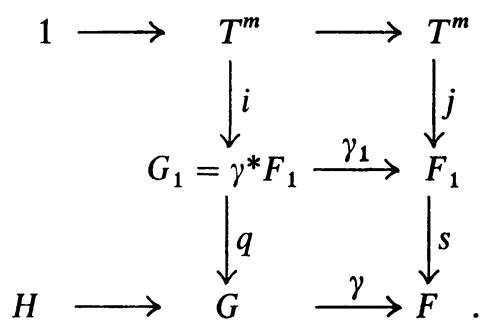

We claim that there is a way of introducing a group operation into $G_{1}=\gamma^{*} F_{1}$ such that $T^{m}$ is a central subgroup and $F_{1}$ is a coset space of $G_{1}$ under the projection $\gamma_{1}$. For this purpose, let us consider the simply-connected covering group $\bar{G}$ of $G$ and let $p: \bar{G} \rightarrow G$ be the covering projection. $\bar{G}$ acts on $G$ and $F$ in obvious ways. It follows from [19] that there exist bundle lifting actions of $\bar{G}$ on $\gamma^{*} F_{1}$ and $F_{1}$ compatible with the bundle mapping $\gamma_{1}$. By bundle lifting actions, we mean that the actions of $\bar{G}$ on $\gamma^{*} F_{1}$ and $F_{1}$ commute with the action of $T^{m}$ and they induce the actions of $\bar{G}$ on $G$ and $F$ by the projections. In particular, there is a mapping $\bar{p}: G \rightarrow \gamma^{*} F_{1}$ such that $q \bar{p}=p$. Let $e$ be the identity of $G$. It is well known that $\pi_{1}(G)=p^{-1}(e)$ is a subset of the centre of $G$. We can choose an admissible mapping $i: T^{m} \rightarrow q^{-1}(e)$ such that $i^{-1} \bar{p}: p^{-1}(e) \rightarrow T^{m}$ is a homomorphism. Now, consider the product actions of $\bar{G} \times T^{m}$ on $\gamma^{*} F_{1}$ and $F_{1}$. Clearly, the bundle mapping $\gamma_{1}: \gamma^{*} F_{1} \rightarrow F_{1}$ is equivariant and the subgroup $A=\left\{\left(x^{-1}, i^{-1} \bar{p}(x) \mid x \in p^{-1}(e)\right\}\right.$ of $\bar{G} \times T^{m}$ acts trivially on both $\gamma^{*} F_{1}$ and $F_{1}$. Hence, the actions of $\bar{G} \times T^{m}$ on $\gamma^{*} F_{1}$ and $F_{1}$ are factored through $G_{1}=\bar{G} \times T^{m} / A$. Following A. Shapiro's construction [16], $\gamma^{*} F_{1}$ is actually the underlying space of $G_{1}$ such that $1 \rightarrow T^{m} \stackrel{i}{\rightarrow} G_{1} \stackrel{q}{\rightarrow} G \rightarrow 1$. Because $T^{m}$ is normal in $G_{1}$ which is connected and the automorphism group of $T^{m}$ is discrete, the extension $1 \rightarrow T \stackrel{i}{\rightarrow} G_{1} \stackrel{q}{\rightarrow} G \rightarrow 1$ is central. On the other hand, since the bundle mapping $\gamma_{1}: G_{1}=\gamma^{*} F_{1} \rightarrow F_{1}$ is equivariant, $F_{1}$ is a coset space of $G_{1}$ and the natural projection of $G_{1}$ onto $F_{1}$ is just $\gamma_{1}$. Let $H_{1}$ be the isotropy subgroup of $F$ relative to $G_{1}$. There is a commutative diagram 


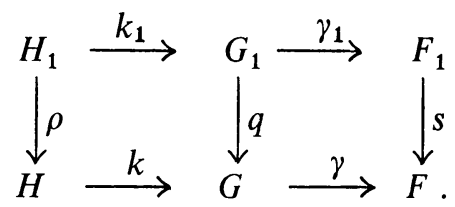

We claim that $\rho$ is an isomorphism, $H_{1} \cap T^{m}=1$, and hence $\left(G_{1} ; T^{m}, H_{1}\right)$ is the triad which we want. First, we prove that $\rho$ is epimorphic. Let $h \in H$. Then, $h$ leaves the base point $b_{0}$ of $F$ fixed. Let $g_{1} \in G_{1}$ such that $q\left(g_{1}\right)=h$. Then, $g_{1}\left(b_{1}\right)$ and $b_{1}$ lie in the same fibre over $b_{0}$ where $b_{1}$ is the base point of $F_{1}$ lying over $b_{0}$. There exists $t \in T^{m}$ such that $\operatorname{tg}_{1}\left(b_{1}\right)=b_{1}$. Clearly, $\rho\left(\operatorname{tg}_{1}\right)=q\left(\operatorname{tg}_{1}\right)=h$. $\rho$ is also monomorphic. Suppose that $\rho\left(h_{1}\right)=\rho\left(h_{2}\right)=h$ for $h_{1}, h_{2} \in H_{1}$. Then $h_{2}=h_{1} t$ and hence $h_{1}, h_{2}$ belong to a single fibre. Since $\gamma_{1}: G_{1} \rightarrow F_{1}$ is a fibre mapping, $\gamma_{1}\left(h_{1}\right)=\gamma_{1}\left(h_{2}\right)$ implies $h_{1}=h_{2}$. In particular, $H \cap T^{m}=1$.

Now, we prove the uniqueness part. Let $\left(G_{1} ; T, H\right)$ and $\left(G_{1}^{\prime} ; T^{\prime}, H^{\prime}\right)$ be two triads such that $T \cong T^{\prime}$ and there exists an equivariant isomorphism $f:\left(F=G_{1} /(T \times H), G=G_{1} / T\right) \rightarrow\left(F^{\prime}=G_{1}^{\prime} / T^{\prime} \times H^{\prime}, G^{\prime}=G_{1}^{\prime} / T^{\prime}\right)$ such that $f^{*} k\left(G_{1}^{\prime} ; T^{\prime}, H^{\prime}\right)=k\left(G_{1} ; T, H\right)$. We are going to show that $\left(G_{1} ; T, H\right)$ is equivalent to $\left(G_{1}^{\prime} ; T, H\right)$. Since $k\left(G_{1} ; T, H\right)=f^{*} k\left(G_{1}^{\prime} ; T^{\prime}, H\right)$ and a principal torus fibration is characterized by its $k$-invariant, we may identify the fibrations $T \rightarrow F_{1} \rightarrow F$ and $T^{\prime} \rightarrow F_{1}^{\prime} \rightarrow F^{\prime}$ of the last columns of the canonical diagrams of the triads $\left(G_{1} ; T, H\right)$ and $\left(G_{1}^{\prime} ; T^{\prime}, H^{\prime}\right)$, respectively. For the middle columns $T^{\stackrel{i}{\rightarrow}} G_{1} \stackrel{q}{\rightarrow} G$ and $T^{\prime} \stackrel{i^{\prime}}{\rightarrow} G_{1}^{\prime} \stackrel{\stackrel{\prime}{\prime}^{\prime}}{\rightarrow} G^{\prime}$ of the canonical diagrams are induced bundles over $G=G^{\prime}$ of the projection $\gamma: G=G^{\prime} \rightarrow F=F^{\prime}$, they are equivalent as bundles. On the other hand, the middle columns are group extensions. Following from A. Shapiro's construction [16], there exist mappings $\bar{p}: \bar{G} \rightarrow \gamma^{*} F_{1}$ and $\bar{p}^{\prime}: \bar{G} \rightarrow \gamma^{*} F_{1}$ such that (i) $q \bar{p}=q \bar{p}^{\prime}=p$, (ii) $i^{-1} \bar{p}: p^{-1}(e) \rightarrow T$ and $i^{-1} \bar{p}^{\prime}: p^{-1}(e) \rightarrow T$ are homomorphisms where $p: \bar{G} \rightarrow G$ is the covering projection, (iii) $G_{1}=(\bar{G} \times T) / A$ and $G_{1}^{\prime}=(\bar{G} \times T) / A^{\prime}$ where $A=\left\{\left(x^{-1}, i^{-1} \bar{p}(x)\right) \mid x \in p^{-1}(e)\right\}$ and $A^{\prime}=\left\{\left(x^{-1}, i^{-1} \bar{p}(x)\right) \mid x \in p^{-1}(e)\right\}$. The natural actions of $\bar{G}$ on $G_{1}$ and $G_{1}^{\prime}$ are the bundle lifting actions on $\gamma^{*} F_{1}$ of the natural action of $\bar{G}$ on $G$ induced by the covering projection $p: \bar{G} \rightarrow G$. Stewart [19] proved that they are equivalent and hence $i^{-1} \bar{p}=i^{-1} \bar{p}^{\prime}$. Again by A. Shapiro's construction [16], there is an isomorphism $h: G_{1} \rightarrow G_{1}^{\prime}$ such that the following diagram is commutative:

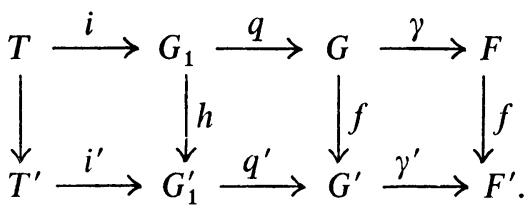

It follows that $h(T \times H)=\gamma^{\prime-1}\left(b_{0}^{\prime}\right)=T^{\prime} \times H^{\prime}$ where $b_{0}^{\prime}$ denotes the base point of $F^{\prime}$ which is understood to be the coset containing the identity of $G_{1}^{\prime}$. Hence, $\left(G_{1} ; T, H\right)$ is equivalent to $\left(G_{1}^{\prime} ; T^{\prime}, H^{\prime}\right)$.

Theorem 2.5 is not true, if $G$ is not semisimple. For example, let $(F, G)=\left(T^{2}, T^{2}\right)$ 
and $T=\mathrm{C}$. Then, there does not exist a triad $\left(G_{1} ; \mathrm{C}, H\right)$ such that $\left(G_{1} ; \mathrm{C}, H\right)$ has $\left(T^{2}, T^{2}\right)$ as its smaller pair and $k\left(G_{1} ; \mathrm{C}, H\right)$ is a nonzero element in $H^{2}\left(T^{2} ; Z\right)$. If there exists a triad $\left(G_{1} ; \mathrm{C}, H\right)$ which has $\left(T^{2}, T^{2}\right)$ as its smaller pair and $k\left(G_{1} ; \mathrm{C}, H\right) \neq 0$, then we have

$$
1 \rightarrow \mathrm{C} \rightarrow G_{1} \rightarrow T^{2} \rightarrow 1 .
$$

By A. Shapiro's theorem [16], $G_{1}$ is the trivial extension of $T^{2}$ by C. So the $k$-invariant of the principal fibration (8) which is $k\left(G_{1} ; \mathrm{C}, H\right)=0$. Hence there does not exist such a triad $\left(G_{1} ; \mathrm{C}, H\right)$.

III. The relationship of the larger pair and the smaller pair of a triad. Now, let us examine the relationship of the larger pair and the smaller pair of a given triad $\left(G_{1} ; T, H\right)$. First we consider the following construction( $\left.{ }^{4}\right)$.

Let $1 \rightarrow T \stackrel{i}{\rightarrow} G_{1} \stackrel{q}{\rightarrow} G \rightarrow 1$ be the middle exact sequence of the canonical diagram (1) of the triad $\left(G_{1} ; T, H\right)$. Following Milnor's construction [12], let $E_{1}$ be the infinite-fold join of $G_{1}$ and $E$ be the infinite-fold join of $G$. Then, $E_{1}$ and $E$ are acyclic spaces such that $G_{1}$ and $G$ act freely on them respectively. Since $T$ is a subgroup of $G_{1}, T$ acts freely on $E_{1}$. Let $E(T)=E_{1}, E\left(G_{1}\right)=E \times E_{1}, E(G)=E$ and let $G_{1}$ act on $E\left(G_{1}\right)=E \times E_{1}$ diagonally. There is a diagram

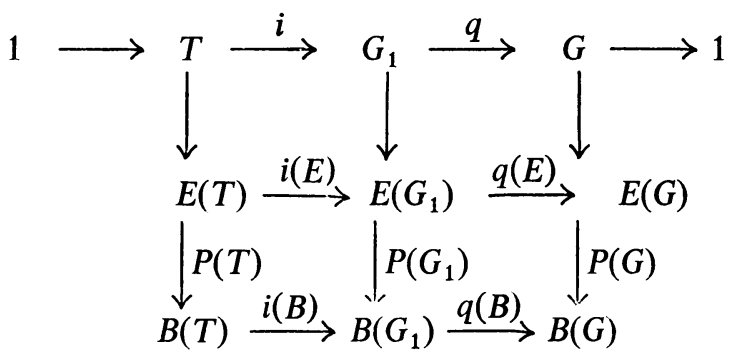

where $B(T)=E(T) / T, B(G)=E\left(G_{1}\right) / G_{1},: B(G)=E(G) / G$. Consider the following bundle:

$$
G_{1} \times T \rightarrow E_{1} \times E(T) \rightarrow\left(E_{1} / G_{1}\right) \times B(T) .
$$

Note that $E(T)$ is just another copy of $E_{1}$, but they have different transformation groups. Since $T$ is a central subgroup of $G_{1}$, the mapping $\mu: G_{1} \times T \rightarrow G_{1}$ which sends $\left(g_{1}, t\right)$ into $g_{1} t$ is a homomorphism and we may consider $G_{1}$ as the quotient group of $G_{1} \times T$ by the central subgroup $T^{\prime}$ consisting of elements of the form $\left(t^{-1}, t\right)$ for $t \in T$. Thus, we have

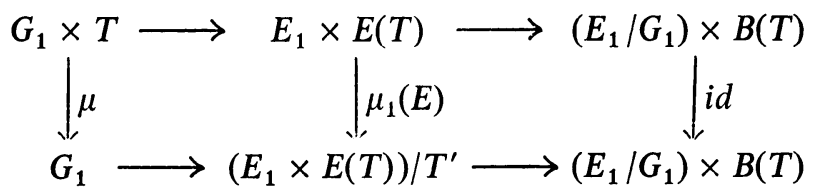

(4) This construction is more or less known. For example, Borel-Hirzebruch [1] vaguely used it when the triad is $(\mathrm{U}(n) ; \mathrm{C}, \mathrm{U}(n-1))$. 
such that $\left(\mu_{1}(E), \mu\right):\left(E_{1} \times E(T), G_{1} \times T\right) \rightarrow\left(\left(E_{1} \times E(T)\right) / T^{\prime}, G_{1}\right)$ is equivariant. Now, since the bottom line of (11) is a $G_{1}$-principal bundle, there exists a bundle mapping

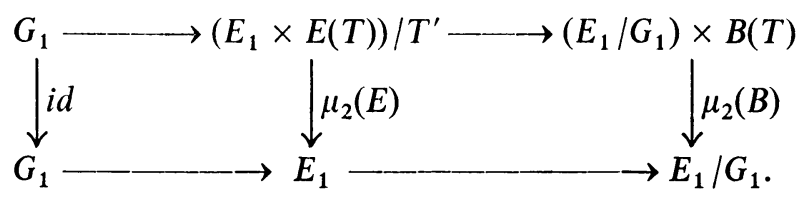

Hence, $\left(\mu_{2}(E), i d\right):\left(\left(E_{1} \times E(T)\right) / T^{\prime}, G_{1}\right) \rightarrow\left(E_{1}, G_{1}\right)$ is equivariant. Let $\bar{\eta}(E)$ $=\mu_{2}(E) \mu_{1}(E)$. The mapping $(\bar{\mu}(E), \mu):\left(E_{1} \times E(T), G_{1} \times T\right) \rightarrow\left(E_{1}, G_{1}\right)$ is clearly equivariant. Now, consider the mapping $\mu(E)=i d \times \bar{\mu}(E): E\left(G_{1}\right) \times E(T)=E \times E_{1}$ $\times E(T) \rightarrow E \times E_{1}=E\left(G_{1}\right)$. It is easy to check that $(\mu(E), \mu):\left(E\left(G_{1}\right) \times E(T), G_{1} \times T\right)$ $\rightarrow\left(E\left(G_{1}\right), G_{1}\right)$ is equivariant. Hence, $\mu(E)$ induces a mapping

$$
\mu(B): B\left(G_{1}\right) \times B(T) \rightarrow B\left(G_{1}\right)
$$

of orbit spaces. It is clear that $\mu(B)$ is the mapping of classifying spaces induced by the homomorphism $\mu: G_{1} \times T \rightarrow G_{1}$.

3.1. THEOREM. (a) Each row and column in (9) is a fibration.

(b) The bottom row $B(T) \stackrel{i(B)}{\longrightarrow} B\left(G_{1}\right) \stackrel{B(q)}{\longrightarrow} B(G)$ of $(9)$ is a principal fibre space with fibre $K\left((Z)^{m}, 2\right)$ and principal mapping $\mu(B): B\left(G_{1}\right) \times B(T) \rightarrow B\left(G_{1}\right)\left({ }^{5}\right)$. (For the definition of principal fibre space, see Peterson-Stein [14].)

(c) Let $\xi \in \mathbf{B}(X ; F, G)$ be induced by $f: X \rightarrow B(G)$.

Then, $\xi$ is weakly associated with a bundle $\eta \in \mathbf{B}\left(X ; F_{1}, G_{1}\right)$ if and only if there exists $h: X \rightarrow B\left(G_{1}\right)$ such that $q(B) h=f$.

Proof. (a) By the way which they were constructed, we know that each column is a universal bundle, the top row and the middle row are obviously fibrations. It remains to show that the bottom line is also a fibration. In fact, locally it is a product. Let $\mathrm{U} \subset B(G)$ such that $p(G)^{-1}(\mathrm{U})$ is homeomorphic to $\mathrm{U} \times G$ compatible with the actions of $G$. We have $q(E)^{-1} p(G)^{-1}(\mathrm{U})$ homeomorphic to $\mathrm{U} \times G \times E(T)$ compatible with the actions of $G_{1}$ which acts diagonally on the last two factors of $\mathrm{U} \times G \times E(T)=\mathrm{U} \times G \times E_{1}$. Hence $p\left(G_{1}\right)$ maps $\mathrm{U} \times G \times E(T) / G_{1}$ homeomorphically onto $q(B)^{-1}(\mathrm{U})$. We note that $G$ acts on $B(T)=E(T) / T=E_{1} / T$ induced by the action $G_{1}$ on $E_{1}$. Hence, the collapsing mapping $(\mathrm{U} \times G \times E(T)) \rightarrow(\mathrm{U} \times G \times E(T)) / G_{1}=q(B)^{-1}(\mathrm{U})$ can be factored into two steps. First, collapse $(\mathrm{U} \times G \times E(T))$ to $\mathrm{U} \times G \times B(T)$ by the action of $T$ on the last factor. Then, collapse $U \times G \times B(T)$ to $(U \times G \times B(T)) / G$ by the diagonal action of $G$ on the last two factors. It is clear that $q(B)^{-1}(\mathrm{U})=(\mathrm{U} \times G \times B(T)) / G$ is homeomorphic to $\mathrm{U} \times B(T)$.

(5) In fact, every fibre space $K(\pi, n) \rightarrow E \rightarrow X$ with simply-connected base space $X$ is fibrehomotopy to a principal $K(\pi, n)$-bundle which is actually nothing but the first stage Postnikov decomposition of the fibre space. (3.1b) claims that the principal map is homotopic to $\mu(B)$. 
(b) It is clear that the fibre of the fibration $B(T) \stackrel{i(B)}{\longrightarrow} B(G)_{1} \stackrel{g(B)}{\longrightarrow} B(G)$ is $K\left((Z)^{m}, 2\right)$. It remains to show that it is a principal fibre space $[14]\left({ }^{5}\right)$ and $\mu(B)$ is the principal map. In other words, we have to show (i) if $i(B): B(T) \rightarrow B(G)$ is the inclusion of the fibre, then $i(B)^{-1} \mu(B)(i(B) \times i d): B(T) \times B(T) \rightarrow B(T)$ defines a homotopically associative multiplication of $B(T)$, (ii) each fibre is (homotopically) invariant under $\mu(B)$. It is easy to verify that $\mu(B)$ satisfies (i) and (ii).

(c) If there is a mapping $h: X \rightarrow B\left(G_{1}\right)$ such that $q(B) h=f$, then it is clear that $\eta=h^{*} v\left(F_{1}, G_{1}\right)$ is weakly associated with $\xi$. Conversely, suppose that there is $\eta \in \mathbf{B}\left(X ; F_{1}, G_{1}\right)$ which is induced by $h^{\prime}: X \rightarrow B\left(G_{1}\right)$ weakly associated with $\xi$. Hence, we have $\left(q(B) h^{\prime}\right)^{*} v(F, G)=f^{*} v(F, G)$. Since $B(G)$ is the classifying space for $(F, G)$-bundles, $q(B) h^{\prime} \sim f$. Because $B(T) \rightarrow B\left(G_{1}\right) \rightarrow B(G)$ is a fibration, we may deform $h^{\prime}$ homotopically into $h$ such that $q(B) h=f$.

Thus we complete the proof of 3.1 .

Since $B(T) \rightarrow B\left(G_{1}\right) \rightarrow B(G)$ is a principal fibre space with fibre $K\left((Z)^{n}, 2\right)$, it is completely determined by its $k$-invariant $\left({ }^{5}\right)$. Before we investigate the relation between this $k$-invariant and $k\left(G_{1} ; T, H\right)$, we introduce a function $\alpha$ which will be used later.

Let $\eta \in \mathbf{B}\left(X ; F_{1}, G_{1}\right)$ be weakly associated with $\xi \in \mathbf{B}(X ; F, G)$ and induced by $h: X \rightarrow B\left(G_{1}\right)$. Let $d \in H^{2}\left(X ;(Z)^{m}\right)$ which determines a mapping $g: X \rightarrow B(T)$ $=K\left((Z)^{m}, 2\right)$ up to homotopy, we define the function

$$
\alpha: H^{2}\left(X ;(Z)^{m}\right) \rightarrow\{\eta \mid \eta \in \mathbf{B}(X ; F, G) \text { weakly associated with } \xi\}
$$

by $\alpha(d)=(\mu(B)(h, g))^{*} v(F, G)$. Since $d$ determines $g$ up to homotopy and $q(B) \mu(h, g)=q(B) h=f, \alpha$ is a well-defined function.

\subsection{Proposition. $\alpha$ is onto.}

Proof. If $\eta_{1} \in \mathbf{B}\left(X ; F_{1}, G_{1}\right)$ is a bundle weakly associated with $\xi$ and induced by a mapping $h_{1}: X \rightarrow B\left(G_{1}\right)$, then $q(B) h_{1} \sim f$. We may assume that $q(B) h_{1}=f$ without loss of any generality. Since $B(T) \rightarrow B\left(G_{1}\right) \rightarrow B(G)$ is a principal fibre space with fibre $K\left((Z)^{m}, 2\right)=B(T)$, there is a mapping $g: X \rightarrow\left((Z)^{m}, 2\right)$ such that $\mu(B)(h, g)=h_{1}$. Let $d \in H^{2}\left(X ;(Z)^{m}\right)$ be the element determined by $g: X \rightarrow B(T)$. Then, $\alpha(d)=\eta_{1}$.

IV. Enlarging the fibre and the group of a bundle. The situation is more interesting when the coset space $F_{1}$ of the larger pair of $\left(G_{1} ; T, H\right)$ is 2-connected.

4.1. Proposition. Given a triad $\left(G_{1} ; T, H\right)$, if the coset space $F_{1}$ of its larger pair is 2-connected, then $s^{*}: \pi_{i}\left(F_{1}\right) \rightarrow \pi_{i}(F)$ is an isomorphism for $i \neq 2$ and $\pi_{2}(F)=(Z)^{m}$ where $s: F_{1} \rightarrow F$ is the natural projection and $m$ is the rank of $T$. Moreover, $k\left(G_{1} ; T, H\right)$ is the fundamental class of $F$. 
This proposition follows immediately from the homotopy exact sequence of the fibration $T \rightarrow F_{1} \stackrel{s}{\rightarrow} F$ which is the last column of the canonical diagram of $\left(G_{1} ; T, H\right)$.

4.2. Definition. Let $\left(G_{1} ; T, H\right)$ be a triad such that the coset space $F_{1}$ of its larger pair is 2-connected. We call the larger pair $\left(F_{1}, G_{1}\right)$ of $\left(G_{1} ; T, H\right)$ an enlargement of the smaller pair $(F, G)$ of $\left(G_{1}: T, H\right)$.

If tollows from (2.5) that if $G$ is semisimple, $F$ is a coset space of $G$ and $\pi_{2}(F)$ is the first nonzero homotopy group of $F$ which is a free group of rank $m$, then $(F, G)$ has an enlargement $\left(F_{1}, G_{1}\right)$. For instance, if $G$ is a simply connected, semisimple, compact, connected Lie group and $F$ is a coset space of $G$ relative to a torus $T$ in $G$; then $(F, G)$ has an enlargement $\left(F_{1}, G_{1}\right)$.

If $\left(F_{1}^{\prime}, G_{1}^{\prime}\right)$ is another enlargement of $(F, G)$, then there exists the following commutative diagram:

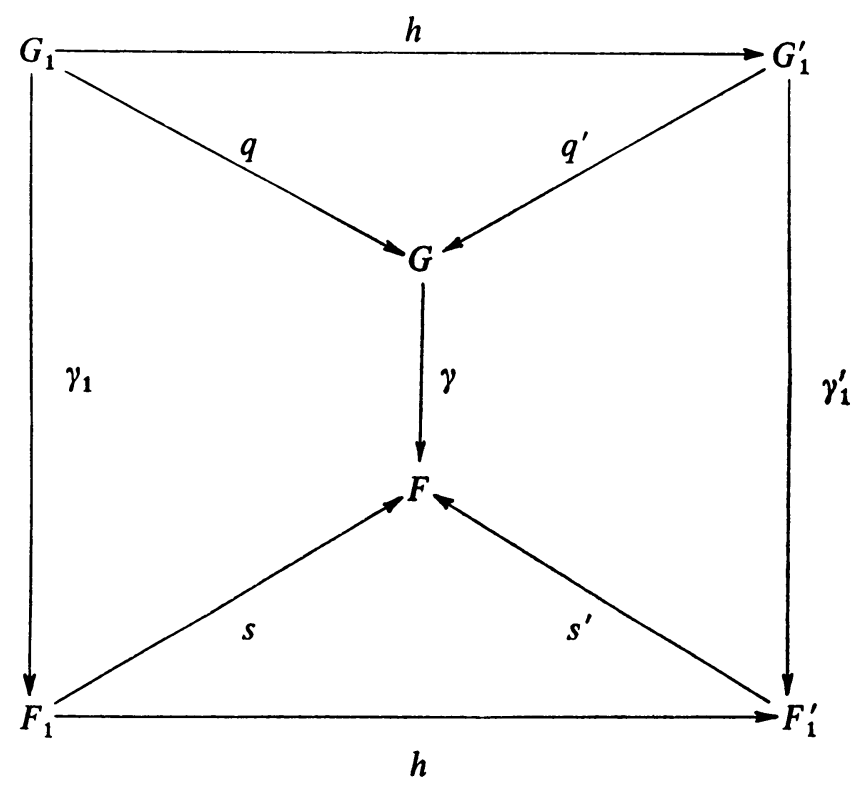

where $h:\left(F_{1}, G_{1}\right) \rightarrow\left(F_{1}^{\prime}, G_{1}^{\prime}\right)$ is an equivariant isomorphism.

4.3. TheOREM. Let $\left(F_{1}, G_{1}\right)$ be an enlargement of $(F, G)$ and $H^{1}(G, z)=0\left(^{6}\right)$.

(a) The primary obstruction to sectioning a bundle $\xi \in \mathbf{B}(X ; F, G)$ is zero if and only if $\xi$ is weakly associated with a bundle $\zeta \in \mathrm{B}\left(X ; F_{1}, G_{1}\right)$.

(b) If $\zeta$ is an $\left(F_{1}, G_{1}\right)$-bundle weakly associated with $\xi$ and if $p: E(\zeta) \rightarrow E(\xi)$ is the natural projection, any cross-section over the nth skeleton of $X$ $c: X^{n} \rightarrow E(\zeta)$ in $\zeta$ yields a cross-section $p c: X^{n} \rightarrow E(\xi)$ in $\xi$. Conversely, if the primary obstruction to sectioning $\xi$ is zero, then for any cross-section

(6) If $G$ is semisimple, $H^{1}(G, Z)$ is automatically zero. 
$\bar{c}: X^{n} \rightarrow E(\xi)(n \geqq 3)$ there exists a bundle $\zeta \in \mathbf{B}\left(X ; F_{1}, G_{1}\right)$ weakly associated with $\xi$ and a cross-section $c: X^{n} \rightarrow E(\zeta)$ such that $p c=\bar{c}$ where $p: E(\zeta) \rightarrow E(\zeta)$ is the natural projection.

Using this theorem, we can reduce the problem of computing obstructions to sectioning a given $(F, G)$-bundle to the problem of lower order obstructions to sectioning the weakly associated $\left(F_{1}, G_{1}\right)$-bundles.

Proof of 4.3. (a) First, we claim that the following diagram

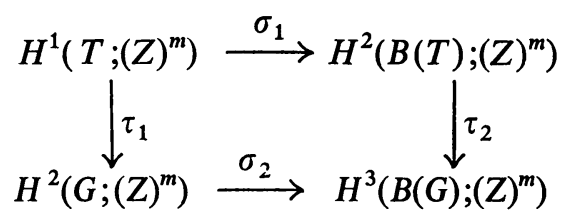

is anti-commutative, i.e., $\tau_{2} \sigma_{1}=-\sigma_{2} \tau_{1}$ where $\sigma_{1}, \sigma_{2}, \tau_{1}, \tau_{2}$ denote the transgressions of $v(T), v(G), q: G_{1} \rightarrow G$ and $q(B): B\left(G_{1}\right) \rightarrow B(G)$ of (9), respectively. Since $H^{1}(G ; Z)=0, H^{1}\left(G ;(Z)^{m}\right)=0$. Hence the groups $E_{2}^{1, q}$ and $E_{2}^{p, 1}$ of the spectral sequence associated with the universal bundle $v(G)$ with coefficients in $(Z)^{m}$ are zero. So, $\sigma_{2}$ is defined and single-valued. It is well known that $\sigma_{1}, \tau_{1}, \tau_{2}$ are also defined and single-valued. It follows from (9) that we have the following commutative diagram of cochain complexes with coefficients in $(Z)^{m}$ :

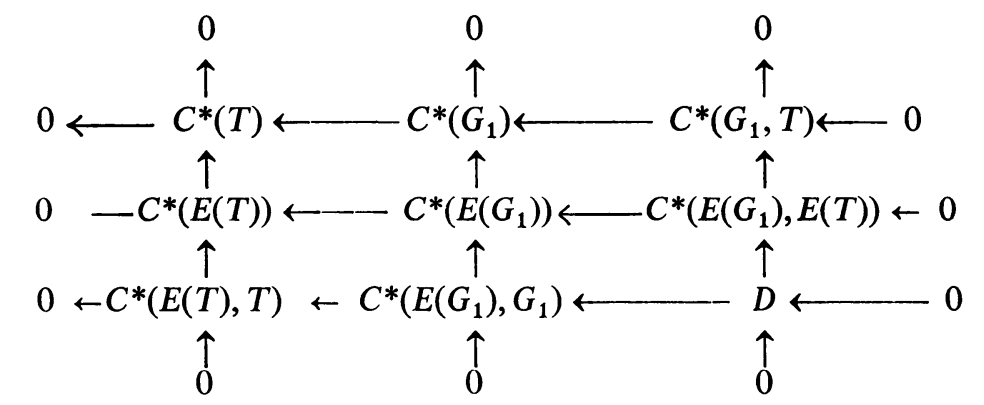

where $D=\operatorname{ker}\left(C^{*}(E(G), G) \rightarrow C^{*}(E(T), T)\right)=\operatorname{ker}\left(C^{*}\left(E\left(G_{1}\right), E(T)\right) \rightarrow C^{*}(G, T)\right)$. Let $\delta_{1}, \delta_{2}, d_{1}, d_{2}$ be the connecting homomorphisms of the top row, the bottom row, the left column and the right column, respectively. It is well known [2] that $d_{2} \delta_{1}=-\delta_{2} d_{1}$. For the dimensions shown in (15), $\delta_{1}, \delta_{2}, d_{1}, d_{2}$ are equivalent to $\tau_{1}, \tau_{2}, \sigma_{1}, \sigma_{2}$, respectively. Therefore, $\tau_{2} \sigma_{1}=-\sigma_{2} \tau_{1}$. Now, if $\xi$ is weakly associated with a bundle $\zeta \in \mathbf{B}\left(X ; F_{1}, G_{1}\right)$, then there is a bundle mapping $p: \zeta \rightarrow \xi$. Since $\pi_{2}\left(F_{1}\right)=0$, there is a cross-section $c: X^{3} \rightarrow E(\zeta)$. Clearly, $p c: X^{3} \rightarrow E(\xi)$ is a cross-section and the primary obstruction to sectioning $\xi$ is zero. Conversely, let $\xi \in \mathbf{B}(X ; F, G)$ be a bundle induced by $f: X \rightarrow B(G)$ such that the primary obstruction vanishes. The only obstruction to lifting $f$ to a mapping $h: X \rightarrow B\left(G_{1}\right)$ is given by

$$
f^{*} \tau_{2}\left(\iota_{2}\right)=f^{*} \tau_{2} \sigma_{1}\left(\iota_{1}\right)=-f^{*} \sigma_{2} \tau_{1}\left(\iota_{1}\right)
$$


where $\iota_{1}, \iota_{2}$ are the fundamental classes of $T$ and $B(T)$, respectively. It follows from the bundle mapping

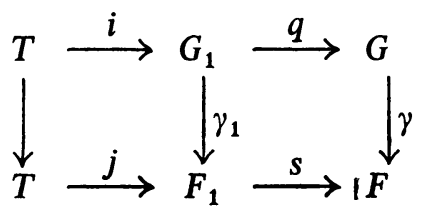

and $\pi_{2}(F)=0$ that $\tau_{1}\left(\iota_{1}\right)=\gamma^{*}(\iota)$ where $\iota$ denotes the fundamental class of $F$. Now, there is a mapping

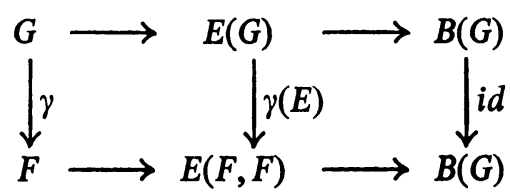

form the universal bundle $v(G)$ onto the universal bundle $v(F, G)$. Hence $\sigma_{3}(\iota)=\sigma_{2} \gamma^{*}(\iota)$ where $\sigma_{3}$ is the transgression of $v(F, G)$. Substituting these relations into (16), we have

$$
f^{*} \tau_{2}(\iota)=-f^{*} \sigma_{3}(\iota)=-f^{*} O_{1}(v(F, G))=-O_{1}(\xi)=0
$$

where $O_{1}(v(F, G))$ and $O_{1}(\xi)$ are the primary obstruction classes of $v(F, G)$ and $\xi$, respectively. Therefore, there is a lifting $h: X \rightarrow B\left(G_{1}\right)$ of $f: X \rightarrow B(G)$ and $h^{*} v\left(F_{1}, G_{1}\right)$ is weakly associated with $\xi$.

(b) The first part is obvious. So, let us prove the second part. First of all, let us pick up one of the $\zeta$ 's, say $\zeta_{1}$, which is weakly associated with $\xi$. Then $p_{1}: E\left(\zeta_{1}\right) \rightarrow E(\xi)$ is a principal $T$-bundle over $\eta$. Consider $\bar{c}^{*} \eta$ which is a principal $T$-bundle over $X^{n}$. Let $O_{1}\left(\bar{c}^{*} \eta\right)$ be the primary and the only obstruction to sectioning $\bar{c}^{*} \eta$ which is an element in $H^{2}\left(X^{n} ;(Z)^{m}\right)$. Since $n>2$, $H^{2}\left(X ;(Z)^{m}\right)=H^{2}\left(X ;(Z)^{m}\right)$ and $O_{1}\left(\bar{c}^{*} \eta\right)$ can be considered as an element in $H^{2}\left(X ;(Z)^{m}\right)$. There exists a unique principal $T$-bundle $\bar{\eta}$ whose $k$-invariant is $-O_{1}\left(\bar{c}^{*} \eta\right)$. Consider the bundle $\zeta_{1} \times \bar{\eta}$ over $X \times X$. Let $d: X \rightarrow X \times X$ be the diagonal embedding: Denote $d^{*}\left(\zeta_{1} \times \bar{\eta}\right)$ by $\zeta_{1} \oplus \bar{\eta}$ which is an $\left(F_{1} \times T, G_{1} \times T\right)$ bundle over $X$. Recall the homomorphism $\mu: G_{1} \times T \rightarrow G_{1}$ which sends $\left(g_{1}, t\right)$ to $g_{1} t$. This induces a mapping $\bar{\eta}: F_{1} \times T \rightarrow F_{1}$ such that $(\bar{\mu}, \mu):\left(F_{1} \times T, G_{1} \times T\right) \rightarrow\left(F_{1}, G_{1}\right)$ is equivariant. Therefore, there is an $\left(F_{1}, G_{1}\right)$-bundle $\zeta_{2}$ weakly associated with $\zeta_{1} \oplus \eta$. It follows from the commutative diagram
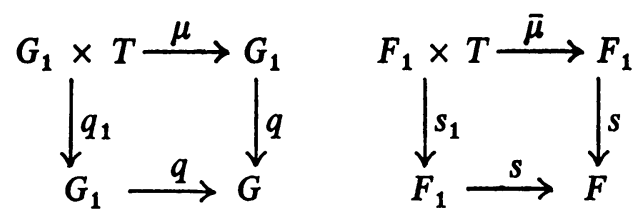

where $q_{1}$ and $s_{1}$ are projections onto the first factors that $\zeta_{2}$ is also weakly 
associated with $\xi$. Consider the principal $T$-bundle $p_{2}: E\left(\zeta_{2}\right) \rightarrow E(\xi)$ which is denoted by $\eta^{\prime}$. It is easy to check that $O_{1}\left(\bar{c}^{*} \eta^{\prime}\right)=O_{1}\left(\eta^{\prime} \mid \bar{c}\left(X^{n}\right)\right)=0$ where $\eta^{\prime} \mid \bar{c}\left(X^{n}\right)$ denotes the portion of $\eta^{\prime}$ over $\bar{c}\left(X^{n}\right)$. Let $c_{2}: \bar{c}\left(X^{n}\right) \rightarrow E\left(\zeta_{2}\right)$ be the cross-section. Clearly, $c_{2} \bar{c}: X^{n} \rightarrow E\left(\zeta_{2}\right)$ is a section in $\zeta_{2}$ and $p_{2} c_{2} \bar{c}=c$. This completes the proof of (b) of 4.3 .

4.4. Corollary. Under the same hypothesis of 4.3, if the base space $X$ of $\xi \in \mathbf{B}(X ; F, G)$ is 2-connected and the primary obstruction to sectioning $\xi$ vanishes, then there is a unique $\eta \in \mathbf{B}\left(X ; F_{1}, G_{1}\right)$ weakly associated with $\xi$ and the $(n+1)$-ary obstruction classes of $\xi$ coincide with the $n$-ary obstruction classes of $\eta$.

Since $H^{2}\left(X ;(Z)^{m}\right)=0$, this corollary follows immediately from 4.3 and 3.2.

V. Kundert formula. Starting with this section, we shall give applications of the previous theorems. The first application is to rederive Kundert's formula for the secondary obstructions to finding a complex line sub-bundle in a given complex vector bundle.

5.1. TheOREM (KUNDERT [7]) $\left({ }^{2}\right) . A \mathrm{CP}^{n-1}$-bundle $\xi \in \mathrm{B}\left(X ; \mathrm{CP}^{n-1}, \mathrm{PU}(n)\right)$ is weakly associated with a bundle $\eta \in \mathrm{B}\left(X ; S^{2 n-1}, \mathrm{U}(n)\right)$ if and only if the primary obstruction $O_{1}(\xi)$ of $\xi$ vanishes.

Now, suppose that $O_{1}(\xi)=0$ and $\eta \in B\left(X ; S^{2 n-1}, \mathrm{U}(n)\right)$ is weakly associated with $\xi$. Then, the secondary obstruction classes to sectioning $\xi$ are given by

$$
\sum_{k=0}^{n} C_{n-k}(\eta)^{\smile D^{k}}
$$

where $D$ varies over $H^{2}(X ; Z), C_{i}(\eta)$ denotes the ith Chern class of $\eta$ and the cup product is taken relative to the standard pairing.

Proof. It was remarked in $\S I I$ that $\left(S^{2 n-1}, \mathrm{U}\right)$ is an enlargement of $\left(\mathrm{CP}^{n-1}, \mathrm{PU}\right)$. Since PU is semisimple, $H^{1}(\mathrm{PU} ; Z)=0$. By 4.3 , the primary obstruction to sectioning $\xi$ vanishes if and only if $\xi$ is weakly associated with a bundle $\eta \in \mathbf{B}\left(X ; S^{2 n-1}, \mathrm{U}\right)$. To prove the second part of the theorem, we first consider the fibration $B(\mathrm{C}) \stackrel{i(B)}{\longrightarrow} B(\mathrm{U}) \stackrel{q(B)}{\longrightarrow} B(\mathrm{PU})$ induced by $1 \rightarrow \mathrm{C} \stackrel{i}{\rightarrow} \mathrm{U} \stackrel{q}{\rightarrow} \mathrm{P} \mathrm{U} \rightarrow 1$. Let $T^{n}$ be the standard maximal torus of $U$. There are the following commutative diagrams:
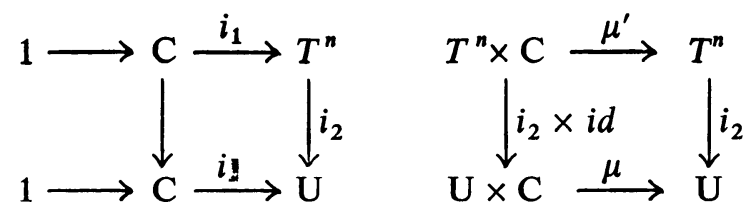

where $i_{1}, i_{2}, i$ are inclusions and $\mu, \mu^{\prime}$ send $(u, c),(t, c)$ to $u c, t c$. They induce the following commutative diagram of classifying spaces: 


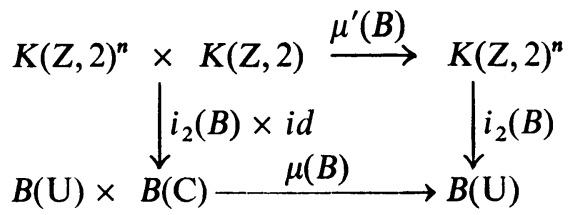

where $\mu(B)$ and $\mu^{\prime}(B)$ are the principal mappings of the fibrations $B(C) \rightarrow B(\mathrm{U}) \rightarrow B(\mathrm{PU})$ and $K(Z, 2) \rightarrow K(Z, 2)^{n} \rightarrow K(Z, 2)^{n-1}=B\left(T^{n} / i_{1} \mathrm{C}\right)$ defined in (13). It is easy to see that $\mu^{\prime}(B)$ is homotopically equivalent to the diagonal action of $K(Z, 2)$ on $K(Z, 2)^{n}$ which sends $\left(k_{1}, \cdots, k_{n} ; k\right)$ to $\left(k_{1} k, \cdots, k_{n} k\right)$. It is well known that $i_{2}(B)^{*}: H^{*}(B(U) ; Z) \rightarrow H^{*}\left(K^{n} ; Z\right)$ is a monomorphism which sends the $i$ th universal Chern class $C_{i}$ into the $i$ th elementary symmetric polynomial $\sigma_{i}$ on the canonical generators $y_{j}(j=1, \cdots, n)$. Since $\mu^{\prime}(B)^{*}\left(y_{j}\right)=y_{j}+d$ where $d$ denotes the fundamental class of the last factor of $K^{n} \times K$, we have that $\mu^{\prime}(B)^{*}\left(\sigma_{i}\right)$ is the $i$ th elementary symmetric polynomial on $y_{j}+d(j=1, \cdots, n)$. By commutativity,

$$
\left(i_{2}(B)^{*} \times 1\right) \mu^{*}(B)\left(C_{n}\right)=\prod_{j=1}^{n}\left(y_{j}+d\right)=\sum_{k=0}^{n} \sigma_{n-k} \times d^{k} .
$$

Since $i_{2}(B)^{*} \times 1$ is monomorphic,

$$
\mu(B) *\left(\mathrm{C}_{n}\right)=\sum_{k=0}^{n} \mathrm{C}_{n-k} \times d^{k}
$$

Now, suppose that $\eta \in \mathbf{B}\left(X ; S^{2 n-1}, \mathrm{U}\right)$ is weakly associated with $\xi$. Let $\eta$ be induced by $h: X \rightarrow B(\mathrm{U})$. By 4.2 and the definition of $\alpha$, any bundle $\eta^{\prime} \in \mathbf{B}\left(X ; S^{2 n-1}, \mathrm{U}\right)$ must be induced by a mapping $(h, g) \mu(B): X \rightarrow B(\mathrm{U})$ where $g: X \rightarrow B(C)=K(Z, 2)$. Hence, it follows from (24) that

$$
\begin{aligned}
\mathrm{C}_{n}\left(\eta^{\prime}\right) & =\sum_{k=0}^{n} \mathrm{C}_{n-k}\left(h^{*} v\right) \smile g^{*}(d)^{k} \\
& =\sum_{k=0}^{n} \mathrm{C}_{n-k}(\eta) \smile g^{*}(d)^{k} .
\end{aligned}
$$

As $g$ varies over the homotopy classes of mappings from $X$ into $B(C), \eta^{\prime}$ varies over the set of $\left(S^{2 n-1}, U\right)$-bundles weakly associated with $\xi$ and $g^{*}(d)$ varies over $H^{2}(X ; Z)$. By 4.3 , the primary obstruction classes of these $\left(S^{2 n-1}, U\right)$ bundles $\eta$ 's weakly associated with $\xi$ are the secondary obstructions of $\xi$. Therefore, $\mathrm{O}_{2}(\xi)$ are given by $(20)$.

VI. Third obstructions to sectioning $\left(\mathrm{CP}^{n-1}, \mathrm{PU}\right)$-bundles. In this section, we shall give a description of the third obstruction classes by applying 4.3 and Liao's formula of the secondary obstructions of sphere bundles $[6 ; 8 ; 9]$. 
6.1. Theorem. Let $\xi \in \mathbf{B}\left(X ; \mathrm{CP}^{n-1}, \mathrm{PU}\right)(n>2)$. If $O_{1}(\xi)=0$ and $O_{2}(\xi)$ contains zero, then there is $\eta \in \mathbf{B}\left(X ; S^{2 n-1}, \mathrm{U}\right)$ weakly associated with $\xi$ and $\mathrm{C}_{n}(\eta)=0$. Moreover, $\mathrm{O}_{3}(\xi)$ is a union of cosets in $\mathrm{H}^{2 n+1}\left(X ; Z_{2}\right)$ relative to the subgroups $\left(S q^{2}+W_{2}(\eta) \smile+n D^{\smile}\right) H^{2 n-1}(X ; Z)$ (one coset for each subgroup) where the cup product is taken relative to the nontrivial pairing and $D$ varies over the elements in $H^{2}(X ; Z)$ satisfying

$$
D^{n}+C_{1}(\eta)^{\smile} D^{n-1}+\cdots+C_{n-1}(\eta)^{\smile} D=0 .
$$

Note that $O_{3}(\xi)$ is not a coset of $H^{2 n+1}\left(X ; Z_{2}\right)$ relative to a single subgroup but a union of cosets relative to some subgroups.

Proof of 6.1. By hypothesis, there is $\eta \in \mathbf{B}\left(X ; S^{2 n-1}, \mathrm{U}\right)$ weakly associated with $\xi$ and a section $c: X^{2 n} \rightarrow E(\eta)$ which lies over $\bar{c}$. Hence, $C_{n}(\eta)=0$. By 4.2, a bundle $\eta^{\prime} \in \mathbf{B}\left(X ; S^{2 n-1}, \mathrm{U}\right)$ weakly associated with $\xi$ can be expressed as $\alpha(D)$ for $D \in H^{2}(X ; z)$. In the proof of 5.1 , we proved that

$$
\mathrm{C}_{n}(\alpha(D))=\sum_{k=0}^{n} \mathrm{C}_{n-k}(\eta)^{\smile} D^{k}=\sum_{k=1}^{n} \mathrm{C}_{n-k}(\eta)^{\smile} D^{k} .
$$

Analogous to the proof of the above formula, we can easily prove that

$$
\mathrm{C}_{1}(\alpha(D))=n D+\mathrm{C}_{1}(\eta)
$$

By 4.3 , the cross-sections in $\xi$ defined on $X^{2 n}$ are always covered by those in $\alpha(D)$ 's for which the cross-sections on $X^{2 n}$ exist. $\alpha(D)$ has cross-sections on $X^{2 n}$ if and only if $\mathrm{C}_{n}(\alpha(D))=\sum_{k=1}^{n} \mathrm{C}_{n-k}(\eta)^{\smile} D^{k}=0$ which is (26). If $\mathrm{C}_{n}(\alpha(D))=0$, then the secondary obstruction classes of its sections on $X^{2 n}$ form a coset of $H^{2 n+1}\left(X ; Z_{2}\right)$ relative to $\left(S q^{2}+W_{2}(\alpha(D) \smile) H^{2 n-1}(X ; Z)\right.$ by Liao's formula [8] where the cup product is taken relative to the nontrivial pairing $Z_{2} \otimes Z \rightarrow Z_{2}$ and $W_{2}(\alpha(D))$ is the second Stiefel-Whitney class of $\alpha(D)$ which is $C_{1}(\alpha(D))$ reduced to mod2. Since $C_{1}(\alpha(D))=n D+C_{1}(\eta)$, the theorem follows immediately.

Now, suppose that $\xi \in \mathbf{B}\left(X ; \mathrm{CP}^{n-1}, \mathrm{PU}\right)$ is the product bundle. Then crosssections in $\xi$ correspond to graphs of the mappings of $X$ into $\mathrm{CP}^{n-1}$. Suppose that $f: X^{2 n} \rightarrow \mathrm{CP}^{n-1}$ is a mapping defined on the $2 n$-skeleton of $X$ into $\mathrm{CP}^{n-1}$. The obstruction to extending $f$ is a cocycle of $X$ with coefficients in $\pi_{2 n}\left(\mathrm{CP}^{n-1}\right)$. If we keep $f: X^{2 n-2} \rightarrow \mathrm{CP}^{n-1}$ fixed and retreat the mapping on $X^{2 n}-X^{2 n-2}$, these cocycles form a set of cohomology classes in $H^{2^{n}+1}\left(X ; Z_{2}\right)$ which is called $\Psi(f)$ by Peterson-Stein [15]. They proved that $\Psi(f)$ is a coset of $H^{2 n+1}\left(X ; Z_{2}\right)$ relative to the subgroup $\left(S q^{2}+f^{*}(\iota)^{-}\right) H^{2 n-1}(X ; Z)$ where the cup product is taken relative to the Whitehead product pairing

$$
\pi_{2 n-1}\left(\mathrm{CP}^{n-1}\right) \otimes \pi_{2}\left(\mathrm{CP}^{n-1}\right) \rightarrow \pi_{2 n}\left(\mathrm{CP}^{n-1}\right)
$$

and $\iota$ denotes the fundamental class of $\mathrm{CP}^{n-1}$. But, if we consider the product bundle $X \times S^{2 n-1}$ as the bundle $\eta$ in (6.1) and the graph of $f$ as cross-section 
of the product bundle $X \times \mathrm{CP}^{n-1}$, then $\mathrm{C}_{i}(\eta)=0$ for all $i$ and $f^{*}(\iota)$ is an element in $H^{2}(X ; Z)$ satisfying (26). $f^{*}(\iota)$ determines a bundle $\eta^{\prime} \in \mathbf{B}\left(X ; S^{2 n-1}, \mathrm{U}\right)$ weakly associated with $X \times \mathrm{CP}^{n-1}$ such that the cross-sections in $\eta^{\prime}$ defined up to $X^{2 n}$ exist. Note that $\eta^{\prime}$ is not a product bundle. As we project these cross-sections into $X \times \mathrm{CP}^{n-1}$, we have the graphs of the mappings of $X^{2 n}$ into $\mathrm{CP}^{n-1}$ which coincide with $f$ on $X^{2 n-1}$. Hence it follows from the proof of 6.1 that $\Psi(f)$ is a coset of $H^{2 n+1}\left(X ; Z_{2}\right)$ relative to the subgroup $\left(S q^{2}+n f^{*}(\iota)^{-}\right) H^{2 n-}(X ; Z)$ $(n>2)$ where the cup product is taken relative to the nontrivial pairing.

6.2. Corollary. The Whitehead pairing. $\pi_{2 n-1}\left(\mathrm{CP}^{n-1}\right) \otimes \pi_{2}\left(\mathrm{CP}^{n-1}\right) \rightarrow \pi_{2 n}\left(\mathrm{CP}^{n-1}\right)$ $(n \geqq 3)$ is zero if $n$ is even and nonzero if $n$ is odd.

Next, we consider a simple example showing that $O_{3}(\xi)$ is not always trivial. Let $\tau^{4 k+1}$ be the tangent bundle of $S^{4 k+1}$. The structure group of $\tau^{4 k+1}$ can be reduced to $\mathrm{U}(2 k)$ and $\tau^{4 k+1}=\xi_{4 k}+\theta$ such that $\mathrm{U}(2 k)$ acts on the fibre $R^{4 k}$ of $\xi_{4 k}$ only and $\theta$ is the trivial bundle. Consider the $\left(\mathrm{CP}^{2 k-1}, \mathrm{PU}\right)$-bundle $\xi$ weakly associated with $\xi_{4 k}$. It is clear that both the primary and secondary constructions vanish in $\xi$. Now, we can consider the associated sphere bundle $\eta$ in $\xi_{4 k}$ as $\eta$ in 6.1. Since $S^{4 k+1}$ is 2 -connected, it follows from 4.4 that $O_{3}(\xi)$ coincides with $O_{2}(\eta) \in H^{4 k+1}\left(S^{4 k+1} ; Z_{2}\right)$. Now, it is well known that $\tau^{4 k+1}$ does not admit an independent 2 -vector field. Hence, $O_{2}(\eta) \neq 0$ which in turn implies that $O_{3}(\xi) \neq 0$.

VII. Secondary obstructions mod 2 to finding orientable 2-plane sub-bundles in orientable vector bundles. In this section, we shall derive formulas for computing the secondary obstructions mod 2 to finding orientable 2-plane sub-bundles in an oriented vector bundle. By an oriented vector bundle, we mean a fibre bundle with $R_{n}$ as the fibre and $\mathrm{SO}(n)$ as the structural group.

7.1. THEOREM. Let $\xi \in \mathbf{B}\left(X ; R^{n}, \mathrm{SO}(n)\right)(n>5)$ be a given orientable vector bundle. Then, the primary obstruction to finding an orientable 2-plane subbundle in $\xi$ is zero and the secondary obstructions $\bmod 2$ are given by

(a) $W_{n-1}(\xi) \in H^{2 n-1}\left(X ; Z_{2}\right)$ for $n$ odd,

(b) $W_{n-1}(\xi)+W_{n-3}(\xi) \smile D+\cdots+W_{3}(\xi) \smile D^{(n-4) / 2} \mathrm{CH}^{2 n-1}\left(X ; Z_{2}\right)$ for $n$ even, where $W_{i}(\xi)$ denotes the ith Stiefel-Whitney class of $\xi, D$ varies over $H^{2}(X ; Z)$ and the cup product is taken relative to the nontrivial pairing.

Proof. First of all, we recall that the obstructions to finding an orientable 2-plane sub-bundle in $\xi$ are equivalent to those to sectioning the bundle $\eta \in \mathbf{B}\left(X ; G_{n-2,2}, \operatorname{PSO}(n)\right)$ (weakly) associated with $\xi$ with oriented Grassmannian Manifold $G_{n-2,2}=\operatorname{SO}(n) /(\operatorname{SO}(n-2) \times \operatorname{SO}(2))$ as the fibre. Since $\operatorname{PSO}(n)$ is semisimple and $\pi_{2}\left(G_{n-2,2}\right)=Z$ is the first nontrivial homotopy group of $G_{n-2,2}$, there is a unique enlargement $\left(F_{1}, G_{1}\right)$ of the fibre pair $\left(G_{n-2}, \operatorname{PSO}(n)\right)$ which 
can be constructed by the method in the proof of 4.3. In fact, we can take $F_{1}=V_{n .2}$, because the principal fibration $\mathrm{SO}(2) \rightarrow V_{n, 2} \rightarrow G_{n-2,2}$ kills off the fundamental class of $G_{n-2,2}$.

Now, we know that $\operatorname{PSO}(n)=\operatorname{SO}(n)$ if and only if $n$ is odd. So, we divide the proof into two cases according to $n$ odd or even.

(a) $n$ (>5) odd. We consider the following commutative diagram:

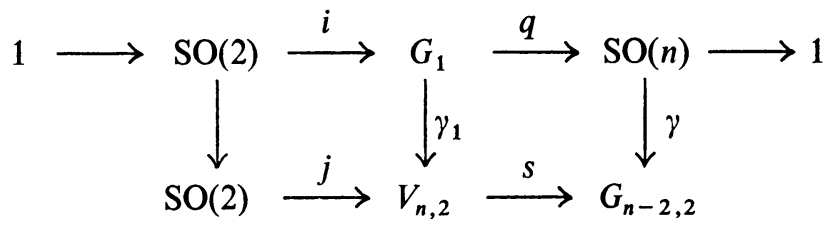

where $i, j$ are inclusions and $q, s, \gamma, \gamma_{1}$ are projections. Since the action of $\operatorname{SO}(n)$ on $G_{n-2,2}$ is factored through $V_{n, 2}, G_{1}=\mathrm{SO}(n) \times \mathrm{SO}(2)$ by the construction in the proof of 4.3 . Hence, the fibration $B(C) \stackrel{i(B)}{\longrightarrow} B\left(G_{1}\right) \stackrel{q(B)}{\longrightarrow} B(\operatorname{SO}(n))$ induced by $1 \rightarrow \mathrm{SO}(2)=\mathrm{C} \stackrel{i}{\rightarrow} G_{1} \stackrel{q}{\rightarrow} \mathrm{SO}(n) \rightarrow 1$ is trivial. In other words, $B\left(G_{1}\right)=B(\mathrm{SO}) \times B(\mathrm{C})$ and the principal mapping $\mu(B): B\left(G_{1}\right) \times B(C) \rightarrow B\left(G_{1}\right)$ is just the multiplication of the second factor. Consider $p: v\left(V_{n, 2}, \mathrm{SO}(n)\right) \rightarrow v\left(G_{n-2,2}, \mathrm{SO}(n)\right)$ where $p$ is the natural projection. Since $\pi_{n-2}\left(V_{n, 2}\right)$ is the first nontrivial homotopy group of $V_{n, 2}$, there exists a section $c: B(\mathrm{SO}(n))^{(n-2)} \rightarrow E\left(V_{n, 2}, \mathrm{SO}(n)\right)$. Obviously, $p c$ : $B(\mathrm{SO})^{(n-2)} \rightarrow E\left(G_{n-2,2}, \mathrm{SO}(n)\right)$ is a cross-section in $v\left(G_{n-2,2}, \mathrm{SO}(n)\right)$. Hence, the primary obstruction to sectioning $v\left(G_{n-2,2}, \mathrm{SO}(n)\right)$ vanishes and $p c$ determines a secondary obstruction class to sectioning $v\left(G_{n-2,2}, \mathrm{SO}(n)\right)$ which is $W_{n-1}$, the $(n-1)$ th Stiefel-Whitney class of the universal vector bundle $v\left(R^{n}, \mathrm{SO}(n)\right)$. Therefore, $q(B)^{*} W_{n-1} \in H^{n-1}\left(B\left(G_{1}\right) ; Z_{2}\right)$ is a secondary obstruction class to sectioning $q(B)^{*} v\left(G_{n-2,2}, \mathrm{SO}(n)\right)$. Because the principal mapping $\mu(B): B\left(G_{1}\right) \times B(\mathrm{C}) \rightarrow B\left(G_{1}\right)$ is the multiplication of the second factor of $B\left(G_{1}\right)$ and $q(B): B\left(G_{1}\right) \rightarrow B(G)$ is the projection of the first factor, we have

$$
\mu(B)^{*} q(B)^{*}\left(W_{n-1}\right)=q(B)^{*}\left(W_{n-1}\right) \times 1 .
$$

This implies that the secondary obstruction class of $\eta \in \mathbf{B}\left(X ; G_{n-2,2}, \mathrm{SO}(n)\right)$ is uniquely determined and is $W_{n-1}(\xi)$ where $\xi \in \mathbf{B}\left(X ; R^{n}, \operatorname{SO}(n)\right)$ is the associated vector bundle which we start with. This completes the proof for $n$ odd.

(b) $n(>5)$ even. This case is more complicated than the first case as one can see from the formula. Set $n=2 m$. $\operatorname{PSO}(2 m)$ is no longer equal to $\operatorname{SO}(2 m)$.

Analogous to the case (a), we have

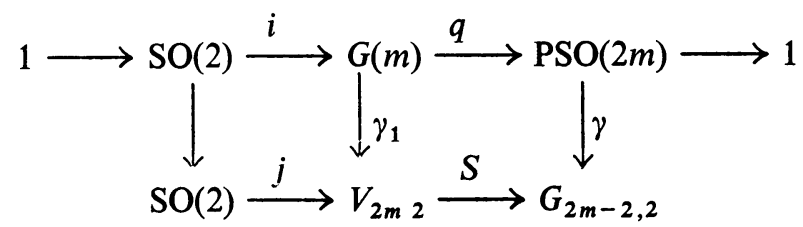


where $G(m)$ can be described as follows. Let $A_{1}$ be the unique subgroup of order 2 in $\mathrm{C}$ and $A_{2}$ the centre of $\mathrm{SO}(2 m)$. Let $A$ be then diagonal subgrouping of $A_{2} \times A_{1} \subset \mathrm{SO}(2 m) \times \mathrm{C}$. Then, $G(m)=(\mathrm{SO}(2 m) \times \mathrm{C}) / A$. It is clear that $G(m)$ contains $\mathrm{SO}(2 m)$ as a normal subgroup. Let $i_{1}: A_{1} \rightarrow \mathrm{C}, i_{2}$ : SO $(2 m) \rightarrow G(m)$ be the inclusions. We have the following commutative diagram of Lie groups:

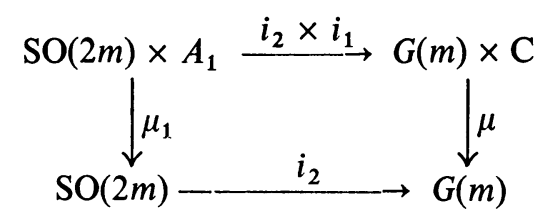

where $\mu, \mu_{1}$ send $(g, e),(s, a)$ to $g e$ and $s a$, respectively. Correspondingly, we have the following commutative diagram of classifying spaces:

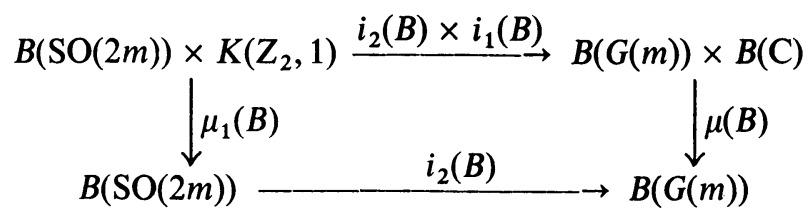

where $\mu_{1}(B), \mu(B)$ are defined in (13). Using an analogous argument as in case (a), we can choose a secondary obstruction class $\mathrm{O}_{2} \in \mathrm{O}_{2}\left(q(B)^{*} v\left(G_{2 m-2,2}, \mathrm{PSO}(2 m)\right)\right.$ such that $i_{2}(B) *\left(O_{2}\right)=W_{2 m-1}$, the $(2 m-1)$ th integer universal Stiefel-Whitney class. Clearly, we have

$$
\left(i_{2}(B)^{*} \times i_{1}(B)^{*}\right) \mu(B)^{*}\left(O_{2}\right)=\mu_{1}(B)^{*} i_{2}(B)^{*}\left(O_{2}\right)=\mu_{1}(B)^{*}\left(W_{2 m-1}\right) .
$$

Let us compute $\mu_{1}(B)^{*}\left(\bar{W}_{2 m-1}\right)$ reduced to $\bmod 2$. In other words, $\mu_{1}(B)^{*}\left(W_{2 m-1}\right)$ where $W_{2 m-1}$ is the $(2 m-1)$ th universal Stiefel-Whitney class $(\bmod 2)$ of $v\left(R^{2 m}, \mathrm{SO}(2 m)\right)$. For this purpose, we consider the following diagram:

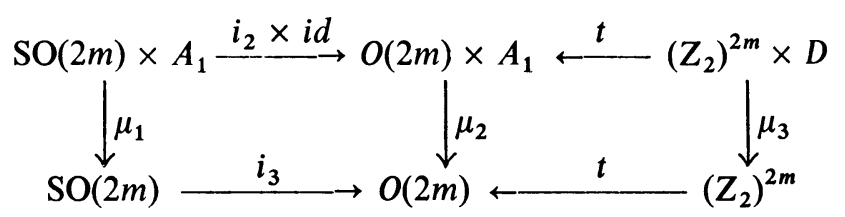

where $t$ maps $\left(Z_{2}\right)^{2 m}$ onto the diagonal orthogonal matrices, $D$ denotes the diagonal subgroup of $\left(Z_{2}\right)^{2 m}$, and $\mu_{i}(a, b)=a b$ for $i=1,2,3$. Corresponding to (36), we have

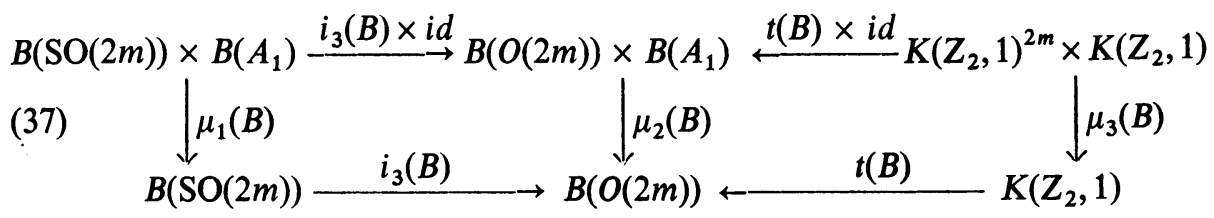


where $\mu_{i}(B)(i=1,2,3)$ are again the mappings defined in (13). It is well known that

$$
i_{3}(B)^{*}: H^{*}\left(B(O(2 m)) ; Z_{2}\right) \rightarrow H^{*}\left(B(\operatorname{SO}(2 m)) ; Z_{2}\right)
$$

is an epimorphism and the kernel is generated by $W_{1}$

$$
t(B)^{*}: H^{*}\left(B(O(2 m)) ; Z_{2}\right) \rightarrow H^{*}\left(K\left(Z_{2}, 1\right)^{2 m} ; Z_{2}\right)
$$

is a monomorphism and $t(B)^{*}\left(W_{i}\right)=\sigma_{i}$ the $i$ th elementary symmetric polynomials on the canonical generators $\left\{x_{1}, \cdots, x_{2 m}\right\}$. By an argument analogous to the proof of 5.1, we have

$$
\mu_{3}(B)^{*}\left(W_{2 m-1}\right)=W_{2 m-1}+W_{2 m-3} \times a^{2}+\cdots+W_{1} \times a^{2 m-2} \bmod 2
$$

where $a$ is the fundamental class of $H^{1}\left(B\left(A_{1}\right) ; Z_{2}\right)$. By the epimorphism $i_{3}(B)^{*}$, we have

$$
\mu_{2}(B)^{*}\left(W_{2 m-1}\right)=W_{2 m-1}+W_{2 m-3} \times a^{2}+\cdots+W_{3} \times a^{2 m-4} \bmod 2 .
$$

Consequently, we have

$$
\mu(B)^{*}\left(O_{2}\right)=\tilde{W}_{2 m-1}+\tilde{W}_{2 m-3} \times d+\cdots+\tilde{W}_{3} \times d^{m-2}
$$

$\bmod 2$

where $\tilde{W}_{2 i-1} \in H^{*}\left(B(G(m)) ; Z_{2}\right)$ such that $i_{2}(B)^{*}\left(\tilde{W}_{2 i-1}\right)=W_{2 i-1}, d$ is the generator of $H^{2}\left(B(C) ; Z_{2}\right)$ and $I$ denotes the ideal generated by $\operatorname{ker} i_{2}(B)^{*} \times 1$.

Now, let $\eta \in \mathrm{B}\left(X ; G_{2 m-2}\right.$, $\left.\mathrm{PSO}(2 m)\right)$ be the bundle weakly associated with $\xi \in \mathbf{B}\left(X ; R^{2 m}, \mathrm{SO}(2 m)\right)$. There is a commutative diagram

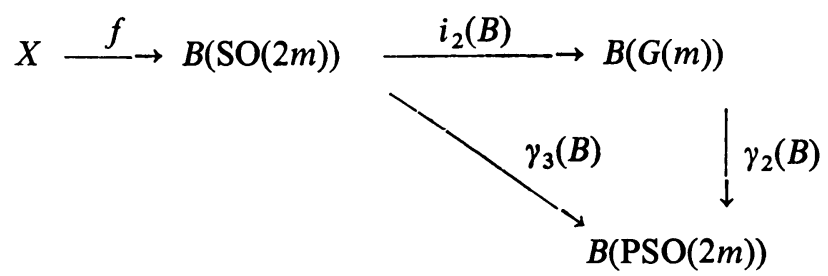

such that $f$ induces $\xi$ and $\gamma_{3}(B) f$ induces $\eta$. Hence, it follows from (40) and (41) that the secondary obstruction classes $\bmod 2$ to sectioning $\eta$ are given by

$$
W_{2 m-1}(\xi)+W_{2 m-3}(\xi) \smile D+\cdots+W_{3}(\xi) \smile D^{m-2}
$$

where $W_{i}(\xi)$ is the $i$ th Stiefel-Whitney class of $\xi, D$ varies over $H^{2}(X ; Z)$ and the cup product is taken relative to the nontrivial pairing $Z_{2} \otimes Z \rightarrow Z_{2}$. This completes the proof for $n$ even.

REMARK. When $n$ is even, the secondary obstruction classes to finding an orientable 2-plane sub-bundle in an oriented vector bundle are integer classes. Since $W_{2 i-1}(\xi)$ are integer classes reduced to $\bmod 2$ and $D$ is also an integer class, 
it is natural to conjecture that the formula (b) of (7.1) is actually a formula for integer classes.

7.2. Proposition. Let $\xi \in \mathbf{B}\left(X ; R^{n}, \mathrm{SO}(n)\right)$ such that $X$ is 2-connected. Then $\xi$ has a 2-plane sub-bundle which is always orientable if and only if $\xi$ has a 2-vector field.

7.2 follows immediately from 4.4 .

Applying 7.2 to the tangent bundles of quaternionic Grassmannian manifolds, we have the following corollary.

7.3. Corollary. Quaternionic Grassmannian manifolds do not have a tangent 2-plane field.

In fact, every quaternionic Grassmannian manifold is 3-connected and has nonzero Euler class. Hence, 7.3 follows immediately from 7.2.

Appendix. Relation between the method of universal models and the method of enlargement. There is a general procedure, called the method of universal models $[6 ; 9 ; 15]$, for studying secondary obstructions to sectioning fibre bundles. First, let us briefly recall this method. Let $B(G)$ be the classifying space of $G$ and $O_{1} \in H^{n+1}\left(B(G) ; \pi_{n}(F)\right)$ be the universal primary obstruction class to sectioning $v(F, G)$. Let $K\left(\pi_{n}(F), n\right) \stackrel{i}{\rightarrow} B(1) \stackrel{q}{\rightarrow} B(G)$ be the principal $K\left(\pi_{n}(F), n\right)$-bundle killing off $O_{1}$. Then the primary obstruction to sectioning $q^{*} v$ is zero. Let $O_{2} \in H^{m+1}\left(B^{1}(G) ; \pi_{m}(F)\right)$ be a secondary obstruction class to sectioning $q^{*} v$. Then, the secondary obstruction classes to sectioning a bundle $\xi \in B(X ; F, G)$ with vanishing primary obstruction are the secondary characteristic classes $[6 ; 15]$ determined by the universal model $\left(B^{1}(G), q^{*} v, O_{2}\right)$. In fact, the fibration $K\left(\pi_{n}(F), n\right) \stackrel{i}{\rightarrow} B^{1}(G) \stackrel{q}{\rightarrow} B(G)$ is just the first stage Postnikov decomposition of $v(F, G)[4 ; 5]$.

Now, let us examine the relation between the method of universal models and the method of enlargement. For convenience, we introduce the following definition.

Definition. Let $J$ be the automorphism of the group $K\left(\pi_{n}(F), n\right)$ which sends each element to its inverse. Then, $J$ induces a bundle involution $\mathrm{j}$ on a principal $K\left(\pi_{n}(F), n\right)$-bundle $\eta$. We say a principal $K\left(\pi_{n}(F), n\right)$-bundle $\eta^{\prime}$ is weakly equivalent to $\eta$ if it is equivalent to $\eta$ or $\mathbf{j} \eta$.

In fact, if we consider $J$ as the generator of an abelian group $Z_{2}$ and $K^{\prime}$ be the split extension $1 \rightarrow K \rightarrow K^{\prime} \rightarrow Z_{2} \rightarrow 1$ determined by $J k J^{-1}=k^{-1}$, then two principal $K\left(\pi_{n}(F), n\right)$-bundles are weakly equivalent if and only if they are equivalent as $K^{0}$-bundles.

Since $B(T) \stackrel{i(B)}{\longrightarrow} B\left(G_{1}\right) \stackrel{q(B)}{\longrightarrow} B(G)$ of (9) is a principal fibre space (cf. Theorem 3.1) with fibre $B(T)$ which is $K\left((Z)^{m}, 2\right)$, it is fibre-homotopy equivalent to its first 
Postnikov decomposition $[4 ; 5]$ which can be constructed as a principal $K\left((Z)^{m}, 2\right)-$ bundle. We still denote this bundle by $B(T) \stackrel{i(B)}{\longrightarrow} B\left(G_{1}\right) \stackrel{q(B)}{\longrightarrow} B(G)$.

Proposition. The principal fibration $K\left((Z)^{m}, 2\right) \stackrel{i}{\rightarrow} B^{1}(G) \stackrel{q}{\rightarrow} B(G)$ which kills off $O_{1}(v(F, G)) \in H^{3}\left(B(G):(Z)^{m}\right)$ is weakly equivalent to the principal fibration $B(T) \stackrel{i(B)}{\longrightarrow} B\left(G_{1}\right) \stackrel{q(B)}{\longrightarrow} B(G)$ of $(9)$.

Proof. The bundle involution $\mathfrak{j}$ sends the $k$-invariant of a principal $K\left(\pi_{n}(F), n\right)$ bundle into its negative. Since it has been shown in the proof of 4.3 that the $k$-invariant of these two fibrations are negatives of one another, they are weakly equivalent to one another.

So, we may identify these two fibrations by changing the sign of the fundamental class of the fibre.

\section{BIBLIOGRAPHY}

1. A. Borel and F. Hirzebruch, Characteristic classes and homogeneous spaces. I, Amer. J. Math. 80 (1958), 458-538.

2. H. Cartan and S. Eilenberg, Homological algebra, Princeton Univ. Press, Princeton, N. J., 1956.

3. R. Hermann, Obstruction theory for fibre spaces, Illinois J. Math. 4 (1960), 9-27.

4. - Secondary obstruction for fibre spaces, Bull. Amer. Math. Soc. 65 (1959), 5-8.

5. F. Hirzebruch, Neue Topologische Methoden der Algebraische Geometrie, Springer, Berlin, 1956.

6. W.-C. Hsiang, Obstructions to sectioning fibre bundles, thesis, Princeton Univ., Princeton, N.J., 1962.

7. E. G. Kundert, Über Schnittfächen in speziellen Faserungen und Felder reeller und komplexer Linienelemente, Ann. of Math. (2) 54 (1951), 215-246.

8. S. D. Liao, On the theory of the obstruction of fibre bundles, Ann. of Math. (2) 60 (1954) 146-191.

9. M. Mahowald, On obstruction theory in orientable fibre bundles, (to appear).

10. W. S. Massey, On the cohomology ring of a sphere bundle, J. Math. Mech. 7 (1958), 265-289.

11. J. W. Milnor, Construction of universal bundles. I, Ann. of Math. (2) 63 (1956), 273-284.

12. - Construction of universal bundles. II, Ann. of Math. (2) 63 (1956), 430-436.

13. - The geometric realization of a semi-simplicial complex, Ann. of Math. (2) 65 (1957), 357-362.

14. F. P. Peterson and N. Stein, Secondary cohomology operations: two formulas, Amer. J. Math. 81 (1959), 281-305.

15. - On sphere bundle with vanishing Euler class, Ann. of Math. (2) 76 (1962), 510-523.

16. A. Shapiro, Group extension of compact connected Lie groups, Ann. of Math. (2) 50 (1949), 581-586.

17. N. E. Steenrod, Topology of fibre bundles, Princeton Univ. Press, Princeton, N. J., 1951.

18. Cohomology operations and obstructions, Colloquium Note, 1957.

19. T. E. Stewart, Lifting group actions in fibre bundles, Ann. of Math. (2) 74 (1961), 192-198.

YALE UNIVERSITY,

New Haven, Connecticut

Princeton UnIVERsity,

Princeton, New Jersey 